\title{
Apport de la technique de fluorescence indirecte au diagnostic immunologique de la cysticercose humaine
}

\author{
par Christian DAO, Jean-Pierre ARNAUD, Jean PETITHORY \\ et Lucien BRUMPT
}

(Collaboration technique: A. Ricour, C. Peltier, F. Géhin)

Institut de Médecine tropicale ( $\mathrm{P}^{r}$ Lucien BRUMPT), 15, rue de l'Ecole de Médecine, F 75006 Paris

\begin{abstract}
Résumé
La technique d'immunofluorescence indirecte au cours de la cysticercose a été étudiée à l'aide de quatre antigènes différents : scolex, cysticerques, anneaux de $T$. saginata et $T$. solium. La sensibilité de la méthode est bonne, les taux varient dans les cas positifs du $1 / 40^{\circ}$ au $1 / 640^{\circ}$. Les sérums positifs correspondent aux cysticercoses récentes, s'accompagnant de signes neurologiques notamment, et aux formes calcifiées généralisées encore évolutives. La sensibilité de l'immunofluorescence est supérieure à celle de l'immunoélectrophorèse.

La spécificité de la méthode semble bonne, si l'on ne retient comme positifs que les sérums donnant une fluorescence au moins égale au $1 / 40^{\circ}$. Il n'y a pas de faux positifs, parmi les sérums témoins, si on emploie comme antigène une coupe d'anneau de $T$. saginata ou $T$. solium. Les quelques faux positifs ont tous été observés sur coupes de scolex d'E. granulosus, dont l'emploi pris isolément est donc déconseillé.

Les réactions croisées ne permettent pas, quel que soit l'antigène utilisé, de distinguer sérologiquement une cysticercose, un kyste hydatique, une échinococcose alvéolaire ou une cénurose.
\end{abstract}


$\mathrm{La}$ technique de l'immunofluorescence indirecte est donc valable pour rattacher au groupe des Cestodes des nodules sous-cutanés ou musculaires, des tumeurs du système nerveux, mais un diagnostic plus précis nécessite la confrontation des données épidémiologiques, cliniques et histologiques.

\section{Summary}

Human cyticercosis studied by the indirect fluorescent technique. - The authors have studied sera from 35 human cysticercosis cases, with the indirect fluorescent technique, using E. granulosus, C. cellulosae, $T$. saginata and $T$. solium as antigens.

The sensibility of this method seems good, the titers of the sera varying in the positive cases from $1 / 40$ to $1 / 640$. The 13 positive cases correspond to recent infestations, particularly when neurologic symptoms are noted; some disseminated calcified cysticercosis give sometimes high titers. Compared with the immunoelectrophoresis, the indirect IF test is more sensitive.

The specificity is quite good when $T$. solium and $T$. saginata are employed as antigens, if the minimum positive titer is $1 / 40$ in the controlled sera. The specificity is not so good on slices of $E$. granulosus with the antihuman polyvalent gammaglobulin fluorescent rabbit serum. Anyway, if it is always possible to recognize sera from parasitoses with the 4 antigens employed, the cross reactions are very important between the Cestode group, and the IF test cannot separate cœnurosis, echinococcosis and cysticercosis. A more precise diagnosis needs then clinical, epidemiological and histological data.

L'homme héberge dans son intestin la forme adulte de Tania solium; le porc est porteur de Cysticercus cellulosae, stade larvaire, qui, atteignant les muscles, donne la ladrerie. L'homme lui-même peut être l'hôte intermédiaire, c'est-à-dire héberger des cysticerques sous la peau, dans les muscles et surtout dans le système nerveux. La gravité de cette dernière localisation et la difficulté de son diagnostic parasitologique expliquent les études qui lui ont été consacrées.

En France, la maladie humaine a disparu, mais des cas sont importés de la Réunion, de Madagascar, du Portugal. Il existe deux périodes dans l'évolution de la maladie. La période précoce correspond à la phase d'installation des cysticerques, qui sont vivants et provoquent une réaction antigénique; la période tardive est marquée par la mort des parasites, qui souvent se calcifient et deviennent décelables radiologiquement, tandis que les anticorps sériques diminuent puis disparaissent. 
La sérologie fait appel à diverses méthodes. La réaction de fixation du complément $(6,8,11)$ recherche les anticorps dans le sérum des malades, et le liquide céphalorachidien où la réaction serait moins sensible $(4,7,12)$. Les réactions de précipitation en milieu liquide sont utilisables malgré des difficultés dues à de fausses précipitations dues à la substance $C(2,3)$, avec quelques faux positifs chez des témoins normaux (10). La méthode d'immunodiffusion simple en gélose et l'immunoélectrophorèse faites avec des extraits de cysticerques, de $T$. solium et de $T$. saginata adultes ont donné des résultats spécifiques ; mais la sensibilité semble assez faible (9): $20 \%$ des cysticercoses récentes et toutes les formes anciennes calcifiées ne donnent aucun trait de précipitation. Il existe des réactions croisées avec les sérums de malades atteints de parasitoses dues à d'autres Taenias (Cestodes) : kyste hydatique, echinococcose alvéolaire, cénurose.

Nous avons voulu savoir si la technique de fluorescence indirecte peut améliorer la sensibilité et la spécificité du diagnostic immunologique de la cysticercose.

\section{Matériel et méthodes.}

La technique d'immunofluorescence indirecte employée est celle recommandée par P. Ambroise-Thomas (I), en conservant un $\mathrm{pH}$ à 7,2 pour éviter des fluorescences non spécifiques; le conjugué fluorescent a été dilué au $1 / 50^{\circ}$ et le bleu Evans au $1 / 5000^{\circ}$. Le taux de positivité correspond à la plus grande dilution de sérum qui donne encore une fluorescence.

Nous avons utilisé 4 antigènes différents, coupés à une épaisseur de $7 \mu$ et disposés sur lames siliconées: sable hydatique d'Echinococcus granulosus, anneaux de T. solium importés congelés de la Réunion, anneaux de $T$. saginata, Cysticercus cellulosae. Pour chacun de ces 4 antigènes, la lecture de la réaction doit tenir compte de l'emplacement des sites antigéniques spécifiques dans la coupe du parasite. Les anticorps sériques d'un sérum positif viennent se fixer sur ces sites et sont ensuite dévoilés par le conjugué antiglobuline humaine fluorescent. La réaction est positive lorsque l'ensemble de la coupe du parasite présente une vive fluorescence, appréciée comparativement à l'effet obtenu avec un sérum témoin dépourvu d'anticorps. Avec les coupes d'anneaux de T. solium et de T. saginata, les sérums témoins négatifs provoquent une vive fluorescence, très localisée à la cuticule du parasite; le centre de la coupe n'est pas fluorescent et la réaction doit être interprétée comme négative. Avec les scolex d'E. granulosus et $C$. cellulosae, il n'y a pas de fluorescences non spécifiques de la périphérie de la coupe. Pour contrôler la spécificité de la méthode, nous avons ensuite testé les sérums sur des parasites divers: coupes d'abcès amibiens du foie, coupes de douves et bilharzies, de Nématodes.

Les sérums proviennent de 35 sujets atteints de cysticercose étudiés par ailleurs en immunodiffusion radiale et immunoélectrophorèse (9). Pour déceler les réactions croisées, nous avons étudié en immunofluorescence indirecte : 12 sérums d'hydatidose, 4 sérums d'échinococcose alvéolaire et cénurose, 10 taeniases à $T$. saginata, 66 témoins normaux, 16 syphilis et 35 parasitoses diverses. Les dilutions de sérum ont été testées au cours de la même séance sur les divers parasites, pour comparer les taux de positivité vis-à-vis de chacun des antigènes. Un total de 178 sérums a été soumis à la technique 
d'immunofluorescence indirecte, les sérums positifs étant vérifiés systématiquement ; la lecture a été faite par deux observateurs séparément sans connaître le diagnostic parasitologique.

\section{Résultats.}

\section{AU COURS DE LA CYSTICERCOSE.}

\section{A. Sérums de cysticercose et antigènes de différents Cestodes.}

Sur 35 malades atteints de cysticercose, 13 ont un sérum donnant une fluorescence égale ou supérieure au $1 / 40^{\circ}$, 6 ont une positivité faible ou douteuse entre $1 / 20^{\circ}$ et $1 / 40^{\circ}$, 16 sont négatifs au $1 / 20^{\circ}$.

Le tableau I donne la correspondance entre l'aspect clinique de la cysticercose, les résultats obtenus en immunodiffusion et les taux limites obtenus par immunofluorescence sur les 4 antigènes du groupe Cestodes utilisés. Les sérums positifs au-delà du $1 / 40^{\circ}$ correspondent en général aux infestations récentes surtout s'il existe des signes neurologiques. Il faut remarquer que les taux obtenus diffèrent assez peu pour un même malade sur les 4 antigènes, alors qu'on s'attendrait à trouver une positivité plus forte vis-à-vis de l'antigène correspondant, c'est-à-dire les coupes de $C$. cellulosae.

Les formes anciennes calcifiées donnent des réponses douteuses ou négatives. Les 16 sérums négatifs, qui ne figurent pas sur le tableau I, ont été prélevés chez des malades dont la cysticercose avait été reconnue par la découverte fortuite des calcifications caractéristiques, sauf dans un cas opéré 5 ans auparavant d'un unique cysticerque vivant.

B. Sérums de cysticercose et antigènes de familles parasitaires différentes.

Les sérums de cysticercose les plus positifs du tableau I ont été étudiés sur des antigènes différents ; ceci a permis de rechercher si les anticorps sont spécifiques, c'est-àdire incapables de donner une fluorescence indirecte sur d'autres parasites que les coupes de Cestodes.

Le tableau 2 montre les résultats obtenus sur des Nématodes (Dipetalonema vitae ou filaire du mérion), Trématodes (Fasciola hepatica ou grande douve), Schistosoma mansoni (agent de la bilharziose intestinale), Rhizopodes (Entamoeba histolytica dans un abcès hépatique du hamster). Un seul sérum s'est montré positif douteux au $1 / 40^{\circ}$ à la fois vis-à-vis de $D$. vitae et d'E. histolytica; le malade d'origine réunionaise aurait pu posséder des anticorps contre une filaire ( $W$. bancrofti) ou contre $E$. histolytica, car la Réunion est une région d'endémie de ces deux maladies.

II. RECHERCHE de RÉACTIONS CROISÉEs AU COURS DES PARASITOSES A CESTODEs.

\section{A. Sérums d'hydatidose et antigènes de Cestodes.}

Le tableau 3 montre les résultats obtenus sur les mêmes antigènes avec des sérums de malades atteints de kyste hydatique à différentes périodes évolutives. 7 malades sur 12 
Tableau 1. - Comparaison entre le stade évolutif de la cysticercose et la positivité en immunofluorescence et immunodiffusion. Les 16 malades négatifs au $I / 20$ ne figurent pas sur ce tableau.

\begin{tabular}{|c|c|c|c|c|c|c|}
\hline \multirow{2}{*}{$\begin{array}{l}\text { Aspect clinique de } \\
19 \text { cysticercoses séropositives }\end{array}$} & \multicolumn{4}{|c|}{ Taux en I.F. sur: } & \multirow{2}{*}{ 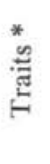 } & \multirow{2}{*}{ 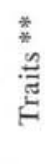 } \\
\hline & $\begin{array}{c}\text { E. gra } \\
\text { nulo- } \\
\text { sus }\end{array}$ & $\begin{array}{l}\text { T. so- } \\
\text { lium }\end{array}$ & $\begin{array}{l}\text { T. sa- } \\
\text { ginata }\end{array}$ & $\begin{array}{l}\text { C.cel- } \\
\text { lulo- } \\
\text { sae }\end{array} \mid$ & & \\
\hline Alb. : cysticercose généralisée calcifiée .. & 80 & 80 & \pm 160 & 80 & 3 & 2 \\
\hline 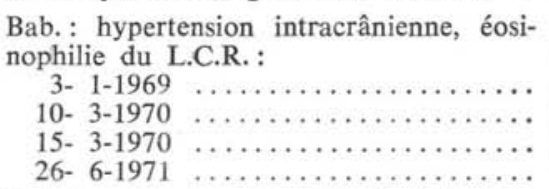 & $\begin{array}{l}80 \\
40 \\
40 \\
40\end{array}$ & $\begin{array}{l}40 \\
20 \\
40 \\
40\end{array}$ & $\begin{array}{l}40 \\
40 \\
40\end{array}$ & $\begin{array}{l}80 \\
40 \\
40\end{array}$ & $\begin{array}{l}2 \\
0 \\
0 \\
0\end{array}$ & $\begin{array}{l}4 \\
0 \\
0 \\
0\end{array}$ \\
\hline Bul. J. : cystic. sous-cutanée évolutive ... & \pm 80 & 20 & \pm 40 & \pm 80 & 0 & 0 \\
\hline Bul. R. : cystic. calcifiée $\ldots \ldots \ldots \ldots \ldots$ & \pm 40 & 40 & \pm 40 & \pm 80 & 0 & 0 \\
\hline Gab.: cysticercose calcifiée $\ldots \ldots \ldots \ldots$ & 20 & 20 & 80 & 20 & 0 & 0 \\
\hline $\begin{array}{l}\text { Las. : cysticercose calcifiée : } \\
8-9-1970 \ldots \ldots \ldots \ldots \ldots \ldots \ldots \ldots \ldots \ldots \ldots \ldots \ldots \ldots \\
27-10-1970 \quad \ldots \ldots \ldots \ldots \ldots \ldots \ldots\end{array}$ & $\begin{array}{l}80 \\
80\end{array}$ & $\begin{array}{l}80 \\
80\end{array}$ & $\begin{array}{l}80 \\
80\end{array}$ & $\begin{array}{l}80 \\
80\end{array}$ & $\begin{array}{l}0 \\
0\end{array}$ & $\begin{array}{l}0 \\
0\end{array}$ \\
\hline Leb. : hypertension intracrânienne ..... & 160 & 80 & 80 & 160 & 2 & 4 \\
\hline $\begin{array}{l}\text { Mal.: âgée de } 3 \text { ans } 1 / 2,2 \text { nodules sous- } \\
\text { cutanés: ablation: }\end{array}$ & & & & & & \\
\hline 29-11-1968 $\ldots \ldots \ldots \ldots \ldots \ldots \ldots \ldots$ & \pm 80 & \pm 80 & \pm 80 & \pm 80 & 2 & 2 \\
\hline 2- 2-1970 $\ldots \ldots \ldots \ldots \ldots \ldots \ldots \ldots$ & 一 & - & - & - & 0 & 0 \\
\hline $22-4-1970 \quad \ldots \ldots \ldots \ldots \ldots \ldots \ldots$ & - & - & 一 & - & 0 & 0 \\
\hline Pal.: cysticercose généralisée calcifiée: & & & & & & \\
\hline 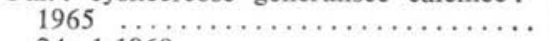 & 160 & 40 & 40 & \pm 160 & 1 & 0 \\
\hline 24-1-1968 $\ldots \ldots \ldots \ldots \ldots \ldots \ldots \ldots$ & 80 & 80 & 40 & \pm 160 & 0 & 0 \\
\hline 18- 8-1968 $\ldots \ldots \ldots \ldots \ldots \ldots \ldots \ldots$ & 160 & 40 & 40 & \pm 160 & & \\
\hline Pay. A. : hypertension intracrânienne ... & 80 & 80 & 40 & 80 & 2 & 2 \\
\hline Pay. E : nombreux cyst. sous-cut. ..... & \pm 160 & 80 & 80 & 160 & 4 & 4 \\
\hline $\begin{array}{l}\text { Péd. : cyst. disséminée et signes neurolo- } \\
\text { giques: }\end{array}$ & & & & & & \\
\hline $1-12-1967 \quad \ldots \ldots \ldots \ldots \ldots \ldots \ldots \ldots$ & 160 & 160 & 160 & 160 & 3 & 4 \\
\hline 18-11-1968 $\ldots \ldots \ldots \ldots \ldots \ldots \ldots \ldots$ & 160 & 160 & 160 & 320 & & \\
\hline $\begin{array}{l}\text { Sad.: syndrome méningé, éosinophilie du } \\
\text { L.C.R. } \cdots \cdots \cdots \cdots \cdots \cdots \cdots \cdots \cdots \cdots \cdots \cdots\end{array}$ & \pm 640 & 320 & \pm 640 & 640 & 8 & 11 \\
\hline Boy. : cystic. généralisée calcifiée $\ldots . .$. . & 20 & \pm 20 & 20 & 一 & 0 & 0 \\
\hline Gas. : cystic. généralisée calcifiée $\ldots . .$. & \pm 40 & & 一 & & 0 & 0 \\
\hline Lon. : 1 cyst. intra-oculaire $\ldots \ldots \ldots \ldots$ & \pm 20 & \pm 20 & - & \pm 20 & 0 & 0 \\
\hline Mal. J. : 2 cyst. sous-cutanés $\ldots \ldots \ldots \ldots$ & - & - & 20 & - & 0 & 0 \\
\hline Pay. G.: cysticercose calcifiée $\ldots \ldots \ldots$ & \pm 20 & \pm 20 & \pm 20 & \pm 20 & & \\
\hline Rig. : 1 cyst. enlevé 3 ans avant $\ldots \ldots$. & 20 & 20 & \pm 40 & \pm 40 & 0 & 0 \\
\hline
\end{tabular}

* Nombre de traits de Ouchterlony, vis-à-vis d'extraits de $T$. solium et $T$. saginata. ** Nombre de traits en immunoélectrophorèse. 
sont franchement positifs, 1 est douteux au $1 / 40^{\circ}$, les 4 autres sont négatifs. Les sept sérums positifs sont ceux de malades atteints de kyste hydatique du foie dans la période pré-opératoire. Le taux de $1 / 40^{\circ}$ a été noté dans le cas FLA... opéré d'un kyste hydatique un an auparavant. Les réactions négatives correspondent aux kystes non antigéniques car le parasite est mort, ou à la disparition des anticorps à distance de l'éxérèse du kyste.

Ainsi, rien ne permet de distinguer d'après ces résultats une cysticercose d'une hydatidose, car il n'y a pas de différence significative des taux observés sur $C$. cellulosae ou sur E. granulosus.

Tableau 2. - Résultats en immunofluorescence de sérums de cysticercoses sur des antigènes parasitaires divers.

\begin{tabular}{|c|c|c|c|}
\hline Antigènes parasitaires & $\begin{array}{l}\mathrm{Nb} \text {. de sérums de } \\
\text { cysticercose }\end{array}$ & positifs & négatifs \\
\hline F. hepatica .......... & 7 & 0 & 7 \\
\hline S. mansoni .......... & 6 & 0 & 6 \\
\hline D. vitae $\ldots \ldots \ldots \ldots$ & 6 & $1\left(1 / 40^{\circ}\right)$ & 5 \\
\hline E. histolytica ........ & 6 & $1\left(1 / 40^{e}\right)$ & 5 \\
\hline
\end{tabular}

Tableau 3. - Résultats obtenus sur antigènes de Cestodes avec des sérums d'hydatidose.

\begin{tabular}{|c|c|c|c|c|c|}
\hline Hydatidoses & Type clinique & $\begin{array}{l}\text { E. gra- } \\
\text { nulosus }\end{array}$ & T. sol. & T. sag. & $\begin{array}{l}\text { C. cellu- } \\
\text { losae }\end{array}$ \\
\hline Bel... ..... & $\begin{array}{l}\text { Kyste hydatique évolutif } \\
\text { du foie. }\end{array}$ & \pm 160 & 40 & \pm 80 & 160 \\
\hline Bun... ...... & $\gg$ & 80 & 40 & \pm 80 & 80 \\
\hline Deb... ...... & $\gg$ & 80 & 20 & 20 & \pm 80 \\
\hline Gha... ...... & $\gg$ & \pm 80 & & \pm 20 & \\
\hline Ken... ...... & » & 320 & 320 & \pm 320 & 640 \\
\hline Reg... ...... & $\gg$ & 160 & 80 & 80 & 160 \\
\hline Vesp........ & 》 & 160 & 80 & 80 & 80 \\
\hline Fla... ........ & $\begin{array}{l}\text { id. } 1 \text { an après interven- } \\
\text { tion. }\end{array}$ & \pm 40 & \pm 40 & \pm 20 & \pm 40 \\
\hline Bra... $\ldots \ldots$ & K.H. calcifié & 一 & - & - & 一 \\
\hline Cah... ...... & K.H. calcifié & 一 & - & 一 & 一 \\
\hline Saa... ....... & K.H. après intervention & 一 & 一 & 一 & 一 \\
\hline Sai... ....... & $\begin{array}{l}\text { K.H. } 10 \text { ans après inter- } \\
\text { vention. }\end{array}$ & 一 & 一 & 一 & 一 \\
\hline
\end{tabular}




\section{B. Autres parasitoses à Cestodes.}

Nous avons pu appliquer la technique d'immunofluorescence indirecte avec les mêmes antigènes à 3 autres parasitoses à Cestodes (tableau 4). Les parasitoses évolutives avec contact intime avec les tissus de l'hôte, comme la cénurose cérébrale, ou l'échinococcose alvéolaire du foie, donnent des réactions franchement positives. Mais, dans ces cas, les réactions croisées sur les 4 antigènes obtenues avec un sérum positif sont telles qu'il n'est pas possible de préjuger au laboratoire de quel Cestode le malade est atteint : il n'y a pas de différence nette entre les taux observés sur les 4 antigènes.

Tableau 4. - Résultats au cours de diverses parasitoses à Cestodes.

\begin{tabular}{|c|c|c|c|c|}
\hline \multirow{2}{*}{ Malades } & \multicolumn{4}{|c|}{ Taux en I.F. sur } \\
\hline & E. granulosus & T. sol. & T. sag. & C. cellulosae \\
\hline Cénurose cérébrale $\ldots \ldots \ldots$ & 40 & \pm 40 & 20 & \\
\hline Echinococcose $1 \ldots \ldots \ldots$ & 160 & \pm 160 & \pm 160 & 160 \\
\hline Echinococcose $2 \ldots \ldots \ldots$ & \pm 160 & 80 & 80 & \pm 160 \\
\hline Echinococcose $3 \ldots \ldots \ldots$ & \pm 160 & 40 & \pm 40 & 80 \\
\hline T. saginata $1 \ldots \ldots \ldots \ldots$ & 40 & 一 & - & 40 \\
\hline$T$. saginata $2 \ldots \ldots \ldots$ & \pm 40 & 20 & - - & - \\
\hline T. saginata 3 à $10 \ldots \ldots$ & 一 & - & - & - \\
\hline
\end{tabular}

Chez les malades porteurs de $T$. saginata, il n'y a pas d'anticorps circulants décelables en règle par cette méthode. Il est vraisemblable que $T$. saginata adulte présent dans la lumière du tube digestif est peu antigénique car son contact avec les tissus de l'hôte est minime.

\section{RECHERCHE DE RÉACTIONS FAUSSEMENT POSITIVES.}

La recherche de réactions faussement positives, réactions correspondant à des anticorps non spécifiques capables de se fixer sur les sites antigéniques des coupes de Ces. todes, a été effectuée avec des sérums de provenances diverses.

Les résultats consignés sur le tableau 5 expliquent que le seuil de sensibilité de la méthode pour rechercher des anticorps de cysticercose soit le $1 / 40^{\circ}$. A cette dilution en effet, aucun des sérums de témoins normaux et aucun des sérums de syphilitiques dont le BW est fortement positif ne donne de fluorescence sur les coupes de scolex ou d'anneaux de Tænia. Le $1 / 20^{\circ}$ pourrait, par contre, donner des difficultés d'interprétation dans $6 \%$ des témoins normaux et $31 \%$ des syphilis sérologiques.

Avec les sérums de malades atteints de parasitoses dont les agents ne sont pas des Cestodes, les réactions faussement positives observées sur les coupes de $T$. saginata et $T$. solium sont l'exception : ainsi un seul sérum sur 117 est positif douteux au $1 / 40^{\circ}$ sur $T$. saginata; il a été prélevé chez un africain polyparasité, porteur d'anticorps fluorescents 
vis-à-vis de la filariose $\left(D\right.$. vitae $\left.1 / 60^{\circ}\right)$ et de la bilharziose $\left(S\right.$. mansoni $\left.1 / 320^{\circ}\right)$. La spécificité des coupes de tænia est donc bonne pour rechercher une parasitose à Cestodes.

Les faux positifs ne sont notés que si on utilise les coupes de scolex d'E. granulosus. Ainsi 19 résultats sur 117 , soit $16,2 \%$ sont difficiles à interpréter : 6 sérums donnent des faux positifs entre $1 / 40^{\circ}$ et $1 / 80^{\circ}$ ( 3 bilharzioses, 2 filarioses, une amibiase hépatique), 13 sérums de parasitoses diverses donnent des positivités limites entre $1 / 20^{\circ}$ et $1 / 40^{\circ}$. Parmi les 4 antigènes de Cestodes employés, la spécificité d'E. granulosus est donc la plus médiocre.

Tableau 5. - Recherche de réactions faussement positives ou douteuses sur des antigènes de Cestodes avec divers sérums.

\begin{tabular}{|c|c|c|c|c|c|}
\hline \multirow{2}{*}{ Sérums utilisés } & & \multicolumn{2}{|c|}{$\begin{array}{l}\text { Nombre de sérums } \\
\text { positifs au } 1 / 40 \text { sur }\end{array}$} & \multicolumn{2}{|c|}{$\begin{array}{l}\text { Nombre de sérums } \\
\text { douteux au } 1 / 40 \text { sur }\end{array}$} \\
\hline & & E. granulosus & $\begin{array}{l}T \text {. sag. et } \\
T \text {. solium }\end{array}$ & E. granulosus & $\begin{array}{l}T \text {. sag. et } \\
T \text {. solium }\end{array}$ \\
\hline Témoins normaux .... & 66 & 0 & 0 & $4(6 \%)$ & 0 \\
\hline Syphilis $(\mathrm{BW}+++)$. & 16 & 0 & 0 & $5(31 \%)$ & 0 \\
\hline Bilharzioses ......... & 18 & $3(16,6 \%)$ & 0 & $3(16,6 \%)$ & $1(5,5 \%)$ \\
\hline Nématodes (divers) $\ldots$. & 10 & $2(20$ & 0 & $1(10 \%)$ & $1(10 \%)$ \\
\hline Amibiase hépatique $\ldots$ & 7 & $1(14$ & 0 & 0 & 0 \\
\hline
\end{tabular}

\section{Discussion.}

La sensibilité de la méthode de fluorescence indirecte est bonne dans les cas récents de cysticercose humaine; les taux varient du $1 / 40^{\circ}$ au $1 / 640^{\circ}$. La sensibilité est plus grande que l'immunoprécipitation en gélose et a permis de trouver des sérums positifs parmi les cysticercoses calcifiées qui n'avaient pas donné d'arc de précipitation par Ouchterlony ou immunoélectrophorèse. Un certain nombre de sérums donnent des positivités faibles, entre $1 / 20^{\circ}$ et $1 / 40^{\circ}$ qui témoignent de la présence d'anticorps, mais à des taux trop faibles pour qu'on puisse les tenir pour significatifs. Ainsi les réactions au $1 / 20^{\circ}$ dans le cas Lon... qui présentait un seul nodule décelable au niveau du globe oculaire.

L'immunofluorescence permet de suivre de façon sensible la décroissance du taux des anticorps à distance de la phase précoce antigénique: les réactions sont négatives deux ans après l'éxérèse de deux cysticerques sous-cutanés dans le cas Mal. N..., douteuse trois ans après l'éxérèse d'un cysticerque dans le cas Rig... Les formes généralisées peuvent mettre des années avant de se négativer, au moins deux ans dans le cas Bab..., au moins trois ans dans le cas Pal... ; certaines formes généralisées donnent encore des réactions positives alors qu'elles sont déjà calcifiées au moment du diagnostic. 
La Spécificité de la technique doit être analysée. Pour un seuil de sensibilité de $1 / 40^{\circ}$, il n'y a pas de réactions faussement positives avec les sérums témoins ni avec les syphilis sérologiques.

Lorsqu'on se sert des coupes de $T$. saginata ou $T$. solium, la spécificité est bonne : aucun sérum n'a donné de faux résultats positifs au-delà du $1 / 40^{\circ}$; l'immunofluorescence indirecte permet alors d'éliminer des parasitoses à Nématodes, Trématodes ou amibes pathogènes. Si on emploie les scolex d'E. granulosus, le pourcentage des faux positifs atteint $5 \%$. L'interprétation est difficile jusqu'au $1 / 80^{\circ}$.

D'après Matossian, il serait possible de continuer à utiliser les coupes de scolex comme antigène, alors que la tendance actuelle est d'y renoncer, il suffirait de ne pas se servir d'un conjugué fluorescent antiglobuline humaine polyvalent car les anticorps non spécifiques seraient de type IgG; la technique serait plus spécifique et permettrait de suivre plus fidèlement l'évolution en employant un conjugué fluorescent monovalent anti $\operatorname{IgM}(5)$.

La spécificité au sein du groupe des Cestodes est mauvaise car les réactions croisées sont très fortes. Un sérum positif à une forte dilution peut correspondre dans notre expérience aussi bien à une cysticercose qu'à un kyste hydatique, une échinococcose alvéolaire ou une cénurose : il n'y a pas d'écart significatif des dilutions vis-à-vis de l'un ou l'autre des 4 antigènes que nous avons étudiés. Dans presque tous les cas, d'ailleurs, la positivité est plus forte d'une dilution sur scolex et cysticerques (antigènes larvaires) que sur coupes d'anneaux de $T$. saginata ou $T$. solium (antigènes adultes). Ceci tient, sans doute, au fait que les anticorps apparaissent lors de la migration dans l'organisme du stade larvaire.

Les réactions croisées incitent à étudier les sérums avec deux antigènes au moins : scolex ou cysticerques plus sensibles mais moins spécifiques, anneaux mûrs un peu moins sensibles mais permettant d'éliminer les réactions croisées. Cette technique est donc applicable quelle que soit la parasitose du groupe Cestode.

\section{REMERCIEMENTS.}

Nous remercions le $\mathrm{D}^{\mathrm{r}} \mathrm{M}$. Jay de l'hôpital psychiatrique de Saint-Paul de nous avoir confié le sérum de ses malades de la Réunion, ainsi que le $T$. soltum congelé.

\section{Bibliographie}

1. Ambroise-Thomas (P.), 1969. - Etude séro-immunologique de dix parasitoses par les techniques d'immunofluorescence. Thèse, Lyon.

2. Biagi (F.) et TAY (J.), 1958. - A precipitation reaction for the diagnosis of cysticercosis. Am. J. trop. med. hyg., 7, 63-65.

3. Biguet (J.), Capron (A.), Tran van Ky (P.), et Roses (F.), 1965. - Présence de substances du type $\mathrm{C}$ dans les antigènes vermineux et de protéine anti $\mathrm{C}$ au cours des helminthiases humaines ou expérimentales. Rev. d'immunologie, 29, 233-240. 
4. Magalhaes (A. E.), 1957. - Reaçao de fixaçao do complemento para cesticercose no liquido cefalorraquidiano. Arq. Neuro-psiq., 15, 183-189.

5. Matossian (R. M.), Kane (G. J.), Chantler (S. M.), Batty (I.) et Sarhadian (H.), 1972. - The specific immunoglobulin in hydatid disease. Immunology, 22, 423-430.

6. Moses (A.), 1911. - Dos metodos biologicos de diagnostico nas cisticercozes. Mem. do Inst. Cewaldo Cruz, 3, 322-326.

7. NiEto (D.), 1956. - Cysticercosis of the nervous system : diagnosis by means of the spinal fluid complement fixation test. Neurology (Mineapolis), 6, 725-738.

8. Pessoa (S. B.) y Fleury da Silveira (G.), 1929. - Sobre a reaçao de complemento e outros de laboratoro para o diagnostico da cysticercose. Sâo Paulo medico, 2, 499517.

9. Petithory (J.), JAY (M.) et Feillet (M.), 1971. - Le diagnostic par immunoélectrophorèse et Ouchterlony de la cyticercose. L'Encéphale, 66, 1-12.

10. Proctor (E. M.), Powell (S. J.) et Elsdon-Dew (R.), 1966. - The serological diagnosis of cysticercosis. Ann. Trop. med. parasit., 66, 146-151.

11. Reis (J. B.), BeI (A.) y Diniz (H. B.), 1949. - Dificuldades no diagnostico diferencial entre cisticercose encefalia e neurolues. Arq. Neuropsiq. (Sâo-Paulo), 7, 156-164.

12. Varleta (J.), Oberhauser (E.) y Weinstein (V.), 1961. - Contribucion al estudio bioquimico de la neurocisticercosis. Bol. chil. de parasit., 16, 62-66. 